\title{
AM-DRIVEN DESIGN OF HYDRAULIC MANIFOLDS: ENHANCING FLUID FLOW AND REDUCING WEIGHT
}

\author{
Yi Zhu*, Shuai Wang, Chao Zhang, Huayong Yang \\ State key laboratory of fluid power and mechatronic systems, Zhejiang University, 38 Zheda Road, 310027 \\ Hangzhou, China \\ "Corresponding author: Tel.: +86 18694580181; E-mail address: yiz@zju.edu.cn
}

\begin{abstract}
Selective laser melting (SLM), one type of metal additive manufacturing (AM) technology, uses a highintensity laser to selectively melt pre-spread metal powders by a layer-on-layer manner. The technology does not only provide a new way of manufacturing but also innovates product design methodology. In this study, a hydraulic block manifold is designed and manufactured using SLM. In this paper, we present an AM-driven design approach of hydraulic manifolds based on a case study. The target is not only to reduce weight but also to enhance fluid flow by optimizing fluid path to reduce pressure drop. The novelty of the research includes developing a design approach of hydraulic manifolds using SLM with a particular focus on fluid flow. Compared to the traditional hydraulic manifold, the weight of the new SLMed hydraulic manifold was reduced by more than $80 \%$, size by half. Pressure loss of the main functional oil circuit was reduced by $31 \%$, illustrating that the new hydraulic manifold design simultaneously achieves lightweight and high performance. This study contributes to providing theoretical guidance to the design of additively manufactured hydraulic components with high performance.
\end{abstract}

Keywords: hydraulic manifold; lightweight; selective laser melting; pressure loss

\section{INTRODUCTION}

Selective laser melting (SLM), one type of metal additive manufacturing (AM) technology, uses a high-intensity laser to selectively melt pre-spread metal powders by a layer-on-layer manner. The technology does not only provide a new way of manufacturing but also innovates product design methodology. Typical examples include AMed fuel nozzles used in GE LEAP engine, heat exchangers, and injection molds with conformal cooling channels. By using AM, hydraulic components can also be compact, lightweight, and high performance, particularly for hydraulic manifolds with complicated fluid passages. However, AM-driven design approach for hydraulic manifolds is lacking which needs to consider supporting structures, building orientation, fluid flow, and wall thickness. Some industrial companies, such as Renishaw, Moog, Aidro, Liebherr, demonstrated their hydraulic manifolds or even integrated components using SLM. Weight and size were considerably reduced. However, detailed data were not published. Schmelzle et al. [1] designed a hydraulic manifold using SLM. 17 parts were combined into a single component and the weight was significantly reduced. In addition, they also proposed a general design approach based on metal AM and the quality inspection was also presented. The main focus is the manufacturability and mechanical properties of the component.

In this paper, we present an AM-driven design approach of hydraulic manifolds based on a case study. The target is not only to reduce weight but also to enhance fluid flow by optimizing fluid path. The novelty of the research includes developing a design approach of hydraulic manifolds using SLM with a particular focus on fluid flows. 


\section{DESIGN}

\subsection{Prototype hydraulic manifold}

Fig. 1 shows a prototype hydraulic manifold that weighs $33 \mathrm{~kg}$ in a missile launch vehicle, the material of which is $316 \mathrm{~L}$ steel and the size of which is $250 \mathrm{~mm} \times 180 \mathrm{~mm} \times 95 \mathrm{~mm}$. There are many ports in the prototype hydraulic manifold, including one oil inlet (red port), one oil outlet (blue port), four control ports (green ports) and seven auxiliary holes (yellow ports). Nine mounting holes that connect with five solenoid directional valves (S1-S5), one check valve, one three-way flow regulator, one pressure relief valve and one pressure sensor guarantee the high controllability of this hydraulic manifold. The rated flow rate of the hydraulic manifold is 15 $\mathrm{L} / \mathrm{min}$ and the rated pressure is $21 \mathrm{MPa}$.

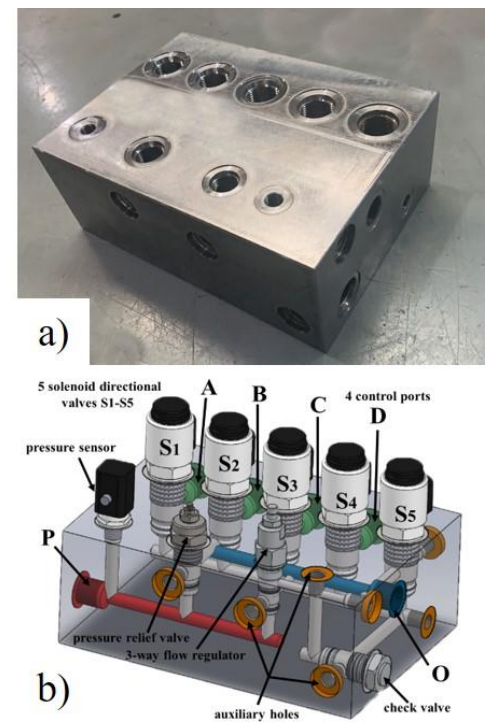

Figure 1: (a) Photograph and (b) schematic diagram of the prototype hydraulic manifold.

\subsection{Design of hydraulic manifold based on AM}

A schematic diagram of the fluid passage logic block extracted from the prototype hydraulic manifold is shown in Fig. 2. It is apparent that the abrupt right-angle turns and regular layout of the fluid passages in the prototype hydraulic manifold are prepared for the convenience of conventional processing, resulting in the bulk shape. The emergence of SLM provides a new advanced technology to fabricate hydraulic manifolds with complex structures without the limitations of traditional processing methods. Thus, the fluid passages could be redesigned to improve the flow performance and reduce the weight. In this work, the local structures and the layout of fluid passages are redesigned.

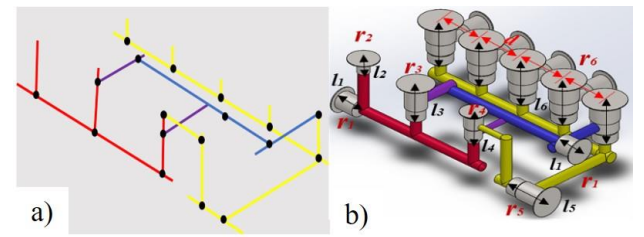

Figure 2: Fluid passage extracted from the prototype hydraulic manifold.

Owing to the limitations of conventional processing, the auxiliary holes are retained in the cross-flow of fluid passages, increasing the risk of leakage and the loss of flow efficiency. Based on the high flexibility of SLM technology, the auxiliary holes can be first removed from the SLMed hydraulic manifold. When hydraulic oils flow in the fluid passages, the abrupt right-angle turns may increase local fluid loss. Replacing these abrupt right-angle turns with gradually curved turns could significantly decrease the loss of flow efficiency. To evaluate the flow efficiency of these three fluid passages with different local designs, a simulation analysis is conducted using computational fluid dynamic software (CFD) software. The accuracy of the CFD analysis results is verified by Zardin et al. [2] using a hydraulic manifold with single passages and theoretical calculations.

The radius of the curved turns $\mathrm{R}$ also has significant effect on the flow efficiency of fluid passages with a diameter $d$. In this simulation, the diameter of all these fluid passages is chosen as $10 \mathrm{~mm}$, which is in accordance with that of the fluid passages in the prototype hydraulic manifold, as shown in Fig. 3(a). Fig. 3(b) shows the effect of the ratio of $R$ to $d$ on the pressure drop between inlet and outlet of a curved fluid passage. It is apparent that the pressure loss decreases as the ratio of $\mathrm{R}$ to $\mathrm{d}$ increases. However, the space is usually limited in the actual layout of fluid passages, and excessive radius of the curved turns will cause interference with adjacent fluid passages. Therefore, the 
design criterion is that the radius of the curved turn should be as large as possible to reduce the pressure loss as much as space permits.

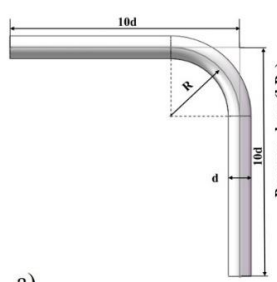

a)

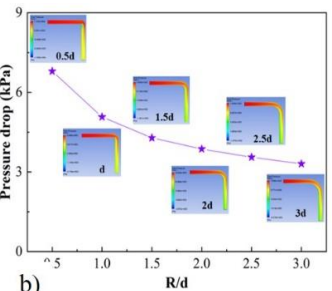

b)

Figure 3: (a) The radius of the curved turns $\mathrm{R}$ and (b) the pressure drop of curved junctions with different radii.

In addition to the local design of fluid passages, a reasonable layout of fluid passages is also a necessary and effective way to design a hydraulic manifold while reducing its weight and improving its flow efficiency. Mitsukiyo et al. [22] indicated that a turn in the flow direction readily causes vortices and flow separation, resulting in greater energy consumption and lower flow efficiency. To obtain a manifold having better performance, many researchers mainly focus on only the arrangement of fluid passages and volume reduction. A design aimed at obtaining the minimum size often implies a rather complicated set of drillings to bring about fluid circulation between external ports of the hydraulic components with higher efficiency loss. Thus, in the rearrangement of fluid passages, the turn numbers of the fluid passages should be minimized as much as possible. Fig. 4 shows the fluid passages from the 3-way flow regulator valve outlet to the solenoid directional valve inlet, and there are six turns in the redesigned hydraulic manifold (as indicated by Model I). In contrast to Model I, Model II replaces two right-angle turns with two obtuseangle turns, and the simulation result of pressure loss for Model II relative to Model I indicates a $13 \%$ reduction. In contrast to the two obtuseangle turns in Model II, the turn number in Model III is further reduced down to two. As a result, compared to that of Model I, the pressure drop of Model III was significantly reduced by $50 \%$. These results indicate that a decrease in the turn numbers of fluid passages can significantly improve the flow efficiency.

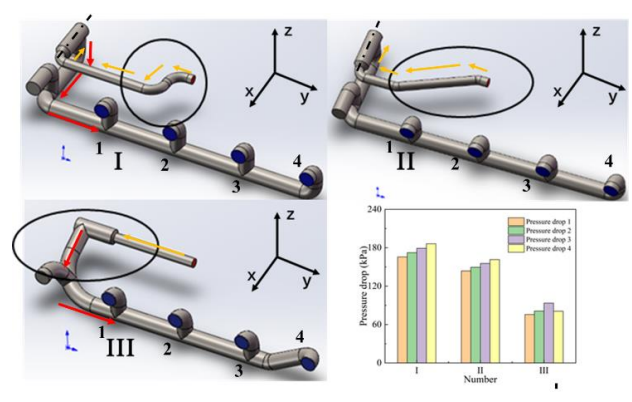

Figure 4: The effect of turn numbers on the pressure drop of fluid passages.

Based on the three aforementioned design criteria, an improved version of a hydraulic manifold with a $3 \mathrm{~mm}$ wall thickness is shown in Fig. 5. It is apparent that compared wo the previous hydraulic manifold, the improved hydraulic manifold has a smaller size of $246 \mathrm{~mm}$ $\times 140 \mathrm{~mm} \times 66.5 \mathrm{~mm}$ without a bulky shell while retaining the original functionality. The layout of the fluid passages is more compact with fewer turns, and the original right-angle turns are replaced by curved turns. Compared with the prototype hydraulic manifold, the new hydraulic manifold had the seven auxiliary holes removed, and the locations of mounting holes that connect with the three-way flow regulator, pressure relief valve and solenoid directional valve S5 were changed to reduce the volume without destroying the function.

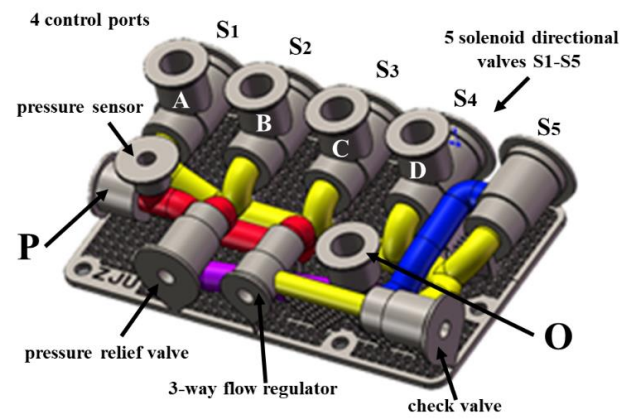

Figure 5: The improved version of the hydraulic manifold 


\section{PROCESS}

The SLM process was conducted in a Renishaw AM 250 machine with an argon atmosphere, which is schematically shown in Fig. 6. The detailed process parameters are indicated in Table 1. After manufacturing, the excess metal powder was removed, and the SLMed hydraulic manifold was obtained from the substrate by a wire electrical discharge machining procedure. The cartridge valve holes and all threaded surfaces were finally improved by machining. A photograph of the finished SLMed hydraulic manifold is shown in Fig. 7.

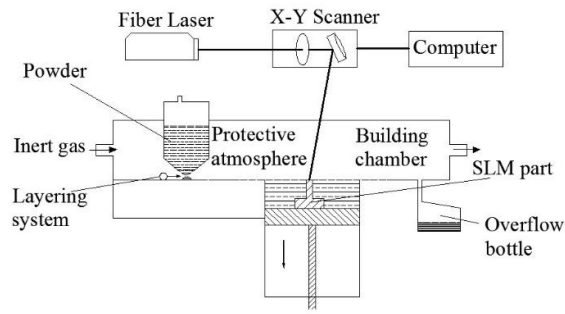

Figure 6: A schematic of SLM system used in the work [3].

Table 1: Process parameters used in the work

\begin{tabular}{|c|c|c|c|c|c|}
\hline 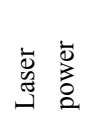 & 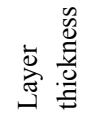 & 芯 & 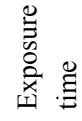 & 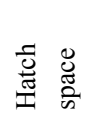 & 总 \\
\hline $200 \mathrm{~W}$ & $50 \mu \mathrm{m}$ & $65 \mu \mathrm{m}$ & $80 \mu \mathrm{s}$ & $80 \mu \mathrm{m}$ & Stripes \\
\hline
\end{tabular}

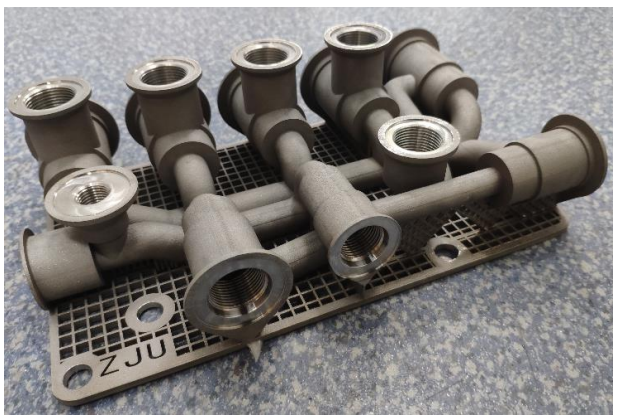

Figure 7: A photograph of the SLMed hydraulic manifold.

\section{INSPECTION AND TESTS}

The forming quality of the SLMed hydraulic manifold, especially the internal structure of the fluid passages, has a significant effect on its working performance. Therefore, it is very important to detect the forming quality of the fluid passages, which cannot be directly observed intuitively without damage. In this study, a 450 $\mathrm{kV}$ industrial CT with micro-and submicrofocus-capable X-ray sources (IPT04013D manufactured by Beijing Granpect Company) was used to inpect the SLMed hydraulic manifold. Fig. 8 shows three-dimensionally visualized maps and local cross-sections of the SLMed hydraulic manifold. It can be found that the structure of the fluid passages is almostly completely in a good shape, indicating the remarkable forming quality. After industrial CT inspection, dynamic cyclical impact tests were used to evaluate the reliability of the SLMed hydraulic manifold. The test result showed that the SLMed hydraulic manifold can continuously work under $21 \mathrm{MPa}$ at $40^{\circ} \mathrm{C}$ for $48 \mathrm{~h}$ without leakage or damage, illustrating that the SLMed hydraulic manifold can satisfy the working requirements achieved by the prototype manifold.

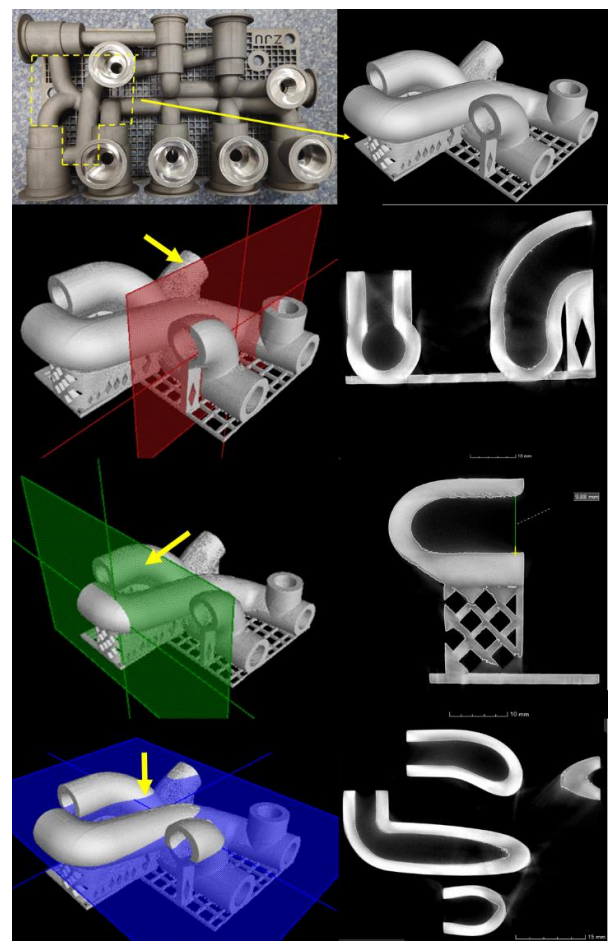

Figure 8: Cross-section of the manifold model and scanning image obtained by industrial CT.

Compared with the $14 \mathrm{~kg}$ prototype hydraulic manifold with a large space size, the SLMed 
hydraulic manifold that weighs $2.6 \mathrm{~kg}$, corresponding to a great weight reduction of up to $80 \%$, and the space size has also been reduced by $46 \%$. In addition to its lightweight, the flow performance of the SLMed hydraulic manifold is also measured and compared to that of the prototype manifold. In this case, experiments and simulations of the flow performance of the main functional oil circuit, i.e., oil circuit 2 , in the prototype and SLMed hydraulic manifolds are conducted. Fig. 9 demonstrates the flow performance tests, while Fig. 10 shows the experimental and simulation results of the flow performance tests for the prototype and SLMed hydraulic manifolds. It is apparent that the experimental results of pressure loss are in good accordance with the simulation results for both the prototype and SLMed hydraulic manifolds. Compared with the prototype hydraulic manifold, the SLMed hydraulic manifold achieved pressure loss reductions of $33 \%, 31 \%, 23 \%$ and $37 \%$ for the port 1 , port 2 , port 3 , and port 4 , respectively, and the average pressure loss reduction of the four ports is $31 \%$. These results indicate that compared to the prototype hydraulic manifold, the SLMed hydraulic manifold achieved better flow performance.

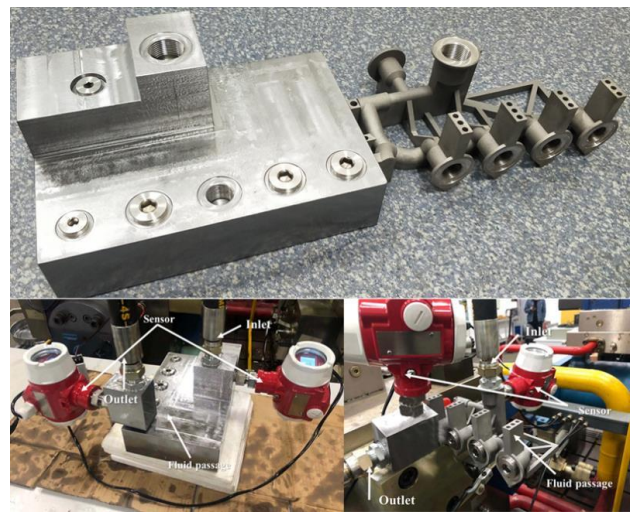

Figure 9: (a) Main functional oil circuit of the prototype (left) and SLMed (right) hydraulic manifolds prepared for flow performance tests; and the flow performance test of (b) prototype and (c) SLMed hydraulic manifolds.

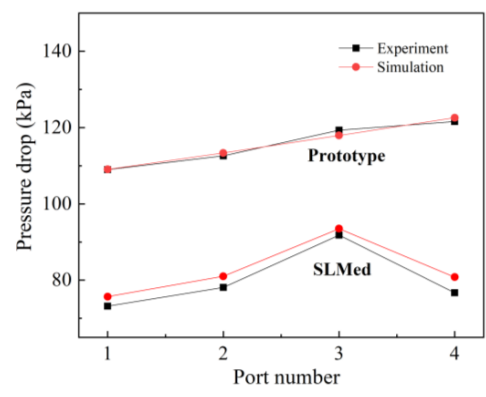

Figure 10: Experimental and simulation results of the flow performance test for both prototype and SLMed hydraulic manifolds.

\section{REFERENCES}

[1] J. Schmelzle, E. V Kline, C.J. Dickman, E.W. Reutzel, G. Jones, T.W. Simpson, (Re)Designing for Part Consolidation: Understanding the Challenges of Metal Additive Manufacturing, Journal of Mechanical Design. 137 (2015) 111404. doi:10.1115/1.4031156.

[2] B. Zardin, G. Cillo, M. Borghi, A. D'Adamo, S. Fontanesi, Pressure losses in multiple-elbow paths and in V-bends of hydraulic manifolds, Energies. 10 (2017). doi:10.3390/en10060788.

[3] Y. Yang, Y. Zhu, M.M. Khonsari, H. Yang, Wear anisotropy of selective laser melted 316L stainless steel, Wear. 428-429 (2019) 376-386. doi:10.1016/j.wear.2019.04.001. 